\title{
Multiple Source Location Estimation Using the EM Algorithm
}

\author{
by
}

Ehud Weinstein and Meir Feder

Woods Hole Oceanographic Institution

Woods Hole, Massachusetts 02543

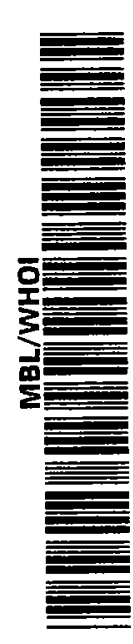

July 1986

Technical Report

Funding provided by the Naval Air Systems Command under contract Number N00014-85-K-0272.

eproduction in whole or in part is permitted for any purpose of the United States government. This report should be cited as:

Woods Hole Oceanog. Inst. Tech. Rept. WHOI-86-26.

Approved for publication; distribution unlimited.

Approved for Distribution:

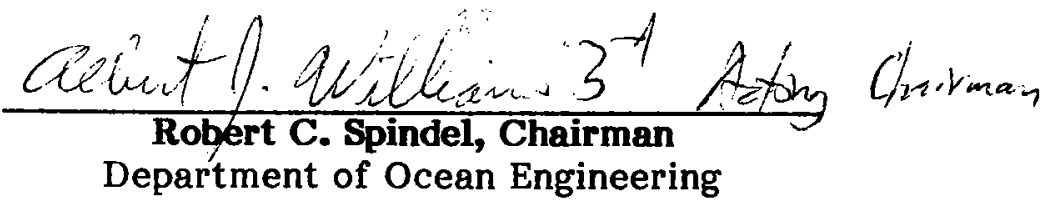


Abstract

We present a computationally efficient scheme for multiple source location estimation based on the EM Algorithm. The proposed scheme is optimal in the sense that it converges iteratively to the exact Maximum Likelihood estimate of all the unknown parameters simultaneously. The method can be applied to a wide range of problems arising in signal and array processing. 


\section{Introduction}

The Maximum Likelihood (ML) method is widely regarded as the optimal procedure in parameter estimation. However, when applied to problems involving. composite signals and a large number of parameters, the ML method tends to be computationally complex and time consuming. As an example, consider the problem of multiple source location estimation by signal observations using an array of spatially distributed sensors. The received signals are composed of the contributions from the various:signal sources, observed in the presence of additive noises. The ML estimate of the various source location parameters jointly requires the solution of a complicated, non-linear optimization problem in several unknowns. Problems of that nature frequently arise in time series analysis, array processing, radar/sonar, acoustical and geophysical signal processing.

In this report we develop a computationally efficient scheme for parameter estimation of composite signals, based on the EM algorithm. The proposed scheme is optimal in the sense that it converges iteratively to the exact ML estimate of all the unknown parameters simultaneously. 
Let $\underline{Y}$ denote the data vector possessing the probability density $f_{\underline{Y}}(\underline{y} ; \underline{\theta})$ indexed by the parameter vector $\underline{\theta} \in \Theta . \Theta$ is a subset of the Euclidean K-space. Given an observed $\underline{y}$, the ML estimate $\underline{\hat{\theta}}_{M L}$ is the value of $\underline{\theta}$ that maximizes the log-likelihood, that is

$$
\underset{\theta<\theta}{\operatorname{Max} \log f_{Y}(y ; \theta) \rightarrow \hat{\theta}_{M L}}
$$

Suppose the data vector $Y$ can be viewed as being incomplete, and we can specify some "complete" data $\underline{X}$ related to $\underline{Y}$ by:

$$
H(X)=\underline{Y}
$$

where $H(\cdot)$ is a non-invertable (many-to-one) transformation. In the multiple source location problem, the 'complete' data could be the observation of the various source signals separately, where the observed (incomplete) data is the sum of the signal contributions from the various sources.

The EM algorithm is directed at finding the solution to (1); however, it does so by making an essential use of the complete data specification. The algorithm is basically an iterative method. It starts with an initial guess $\theta^{(0)}$, and let $\underline{\theta}^{(n+1)}$ be defined inductively by :

$$
\operatorname{Max}_{\underline{\theta}} E\left\{\log f_{\underline{x}}(x ; \underline{\theta}) / \underline{y}=\underline{y} j \underline{\theta}(n)\right\} \longrightarrow \underline{\theta}^{(n+1)}
$$




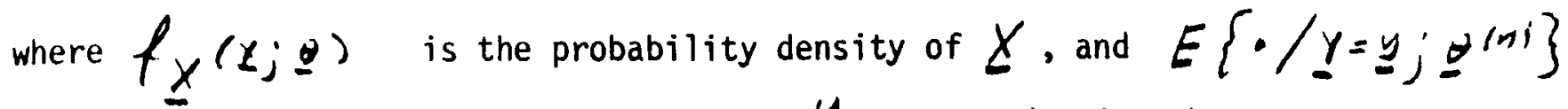
denotes the conditional expectation given $\underline{I}$, computed using the parameter value $\theta^{(n)}$. The heuristic idea here is that we would like to choose $\underline{\theta}$ that maximizes $\log \left(x_{x}(\underline{x} ; \underline{\theta})\right.$, the log-likelihood of the complete data. However, since $\log f_{\underline{x}}(\underline{x} ; \underline{\underline{\alpha})}$ is not available to us (because the complete data is not available), we maximize instead its expectation, given the observed data $\underline{y}$. Since we have used the current estimate $\underline{\theta}^{(n)}$ rather than the actual value of $\boldsymbol{\theta}$ which is unknown, the conditional expectation is not exact. Thus, the algorithm iterates, using each new parameter estimate to improve the conditional expectation on the next iteration cycle and thus to improve the next parameter estimate.

The EM algorithm was first presented in [1], where it is shown that under the usual regularity conditions, the algorithm converges to the desired result, that is $\underline{\theta}(n) \longrightarrow \hat{\theta}_{M L}$, where each iteration increases the likelihood. The basic considerations leading to the EM algorithm (Eq.(3)) are outlined in Appendix $A$ for the convenience of the reader. The rate of convergence of the algorithm is exponential, depending on the fraction of the covariance of the "complete" data that can be predicted using the observed data. If that fraction is small, the rate of convergence tends to be slow, in which case one could use standard numerical methods to accelerate the algorithm.

We note that the EM algorithm is not uniquely defined. The transformation $H($, ) relating $X$ to $\underline{Y}$ can be any non-invertable transformation. Obviously, there are many possible "complete" data specifications that will generate the observed data. Thus, the EM algorithm can be implemented in many possible ways. The final outcome, which is the ML estimate, is completely 
$-4-$

unaffected by the way in which $H(\cdot)$ is specified (i.e., the choice of "complete" data). However, the choice of $H(\cdot)$ may critically affect the complexity and rate of convergence of the algorithm, and the unfortunate choice of $H(\cdot)$ may yield a completely useless algorithm.

We shall proceed as follows: First we develop the EM algorithm for the Linear-Gaussian case. This case covers a wide range of applications. Then we show that for the class of problems of interest here, there is a natural choice of the "complete" data, leading to a surprisingly simple algorithm to extract the ML estimates. 
$-6-$

where $\quad \underline{X}^{(n)} \triangleq E\left\{X / \underline{Y}=\underline{y} ; \underline{\theta}^{(n)}\right\} \quad$ and $\quad \Psi^{(n)} \Delta E\left\{X X^{*} / \underline{Y}=\underline{y} ; \underline{\theta}^{(n)}\right\}$. Since $X$ and $Y$ are jointly Gaussian, these conditional expectations are readily available in the literature (egg. [2], Chap. 5):

$$
\begin{aligned}
& \underline{x}^{(n)}=m(\underline{\theta}(n))+\Gamma(\underline{\theta}(n))[y-H \cdot \underline{M}(\underline{\theta}(n))] \\
& \Psi^{(n)}=[I-\Gamma(\underline{\theta}(n)) \cdot H] \Lambda\left(\underline{\theta}^{(n)}\right)+\underline{x}^{(n)} \underline{x}^{(n) *}
\end{aligned}
$$

where $I$ is the identity matrix, and $\Gamma(Q)$ is the "Kalmar Gain" defined by:

$$
\Gamma(\underline{\theta})=\Lambda(\underline{\theta}) H^{*}\left[H \Lambda(\underline{\theta}) H^{*}\right]^{-1}
$$

The EM algorithm is completely specified by Eqs. (6) - (9); the algorithm iterates between calculating $\underline{X}^{(n)}$ and $\Psi(n)$ and maximizing the expression in (6) with respect to $\theta$, where each iteration increases the likelihood. We observe that $E\left\{\log / \underline{x}(\underline{x} ; q) / Y=y_{j} \theta(n)\right\} \cdot(E q .(6))$ and $\log / x(\underline{x} ; \underline{\theta})$ (Eq. (5)) have the same dependence on $\underline{\theta}$. Maximizing $E\{\log / \underline{x}(\underline{x} ; \underline{\theta)} / \underline{y}=\underline{\underline{y}} ; \underline{\theta}(m)\}$ with respect to $\underline{\theta}$ is the. same as maximizing $\log f_{\underline{x}}(\underline{x} ; \nu)$ with respect to $\theta$. Hence, the EM algorithm essentially requires the $M L$ solution in the $X$ model which might be significantly simpler than the direct $M L$ solution in the $Y$ model. 


\section{Application to Signal Processing}

Let the mathematical model characterizing the observed signal(s)

be given by

$$
\underline{y}(t)=\underline{1}(t ; \underline{\theta})+\underline{n}(t) \quad T_{i} \leq t \leq T_{f}
$$

where $I(t ; \xi)$ are the vector signals received in the absence of noise. We shall be concerned with $I(t ; \vartheta)$ composed of

$$
\underline{I}(t ; \underline{\theta})=\sum_{k=1}^{K} \unlhd_{k}\left(t ; \theta_{k}\right)
$$

where $\theta_{k}$ is the parameter vector associated with the $k$ signal component. The specific problem we have in mind is multiple source location estimation, in which case the $\mathfrak{I}_{k}\left(t ; \mathscr{Q}_{k}\right)$ are the array signals received from the $k$ source, and $\theta_{k}$ are the corresponding source location parameters (bearing and range, velocity components, etc.) In addition, the model given by (10) covers a wide range of problems arising in array and signal processing.

We shall now consider the joint estimation of the various $\theta_{8}$ for the case of deterministic signals and for the case of stochastic Gaussian signals separately.

\section{A. Deterministic Signals}

Consider the model of (10) under the following assumptions:

- The $I_{k}\left(t ; \partial_{k}\right)$ are conditionally known to the observer (i.e., given $\underline{\theta}_{k}$, we can construct $\underline{I}_{k}\left(t ; \underline{\theta}_{k}\right)$ ).

- $\boldsymbol{n}(t)$ are vector zero-mean white Gaussian stochastic processes whose covariance matrix is $E\{\underline{n}(t) \underline{M}(\Delta)\}=Q \cdot \delta(t-\sigma)$, where $Q$ is a constant matrix and $\delta(\tau)$ is the impulse function. 
Under the above assumptions, the log-likelihood function is given by

$\log f_{\underline{y}}\left(\underline{y}_{j} \underline{\theta}\right)=c-\frac{\lambda}{2} \int\left[\underline{y}(t)-\sum_{T_{i}}^{T_{F}} \underline{g}_{k}\left(t ; \underline{\theta}_{k}\right)\right]^{*} Q^{-1}\left[\underline{y}(t)-\sum_{k=1}^{k} \underline{y}_{k}\left(t ; \underline{\theta}_{k}\right)\right] d t$

where $c$ is a normalizing constant. The result in (11) is a straightforward multi-channel extension of the known (deterministic) signal in a white Gaussian noise problem ( [3], Chap. 4). In case we are given only a discrete set of observations $y\left(t_{i}\right) i=1,2, \ldots N$, the log-likelihood is still given by (11), where the integral over $t$ is replaced by the sum over the $t_{i}^{\prime \prime} s$. Thus, the joint ML estimation of the various $\underline{\theta}_{k}^{\prime} \mathcal{s}$ is obtained as the solution to:

$$
\begin{aligned}
& \operatorname{Min}_{\underline{\theta}_{1}, \underline{\theta}_{2}, \ldots \underline{\theta}_{k}}\left\{\int_{T_{i}}\left[\underline{y}(t)-\sum_{k=1}^{T_{f}} \underline{g}_{k}\left(t ; \underline{\theta}_{k}\right)\right]^{*} Q^{-1}\left[\underline{y}(t)-\sum_{b=1}^{K} \underline{J}_{k}\left(t ; \underline{\theta}_{k}\right)\right] d t\right\} \\
& \Longrightarrow \hat{\theta}_{1}, \hat{\theta}_{2}, \ldots \hat{\theta}_{K}
\end{aligned}
$$

In the case of discrete observations, the corresponding optimization problem is:

$$
\begin{gathered}
M_{1 n} \underline{\theta}_{1}, \underline{\theta}_{2}, \ldots \underline{\theta}_{k}\left\{\sum_{i=1}^{N}\left[\underline{y}\left(t_{i}\right)-\sum_{k=1}^{K} \underline{v}_{k}\left(t_{i j} \underline{\theta}_{k}\right)\right]^{*} Q^{-1}\left[\underline{y}\left(t_{i}\right)-\sum_{k=1}^{K} \underline{v}_{k}\left(t_{i j} \underline{\theta}_{k}\right)\right] d t\right\} \\
\longrightarrow \hat{\theta}_{1}, \hat{\theta}_{2}, \ldots \hat{\theta}_{k}
\end{gathered}
$$

In either case, the ML method calls for the optimization of the log-likelinood function with respect to all the $\underline{\theta}_{k}^{\prime} \mathcal{1}$ jointly. Of course, brute force can always be used to solve the problem, evaluating the objective function on a coarse grid to roughly locate the global minimum, and then applying the Gauss method or Newton-Raphson or some other iterative gradient-search algorithm. However, when applied to the problem at hand, these methods tend to be very complex and 
computationally time consuming.

Having the EM algorithm in mind, we would like to simplify the optimization problem associated with the direct ML approach. To apply the algorithm to the problem at hand, the first step is to specify the "complete" data. A natural choice of the "complete" data is obtained by decomposing $y(t)$ into

$$
y(t)=\sum_{k=1}^{k} x_{k}(t)
$$

where

$$
\underline{x}_{k}(t)=\underline{I}_{k}\left(t_{j} \underline{\theta}_{k}\right)+\underline{n}_{k}(t)
$$

and the $\underline{n}_{k}(t)$ are chosen to be mutually uncorrelated, zero-mean Gaussian vector stochastic processes satisfying

$$
\sum_{k=1}^{K} \underline{n}_{k}(t)=\underline{n}(t)
$$

Let the complete data $\underline{X}(t)$ be composed of the various $\underline{X}_{k}(t)$ 's. Then from (14.1), the relation between the "complete" data and the observed (inconplete) data is given by

$$
\underline{y}(t)=H \cdot \underline{x}(t)
$$

where

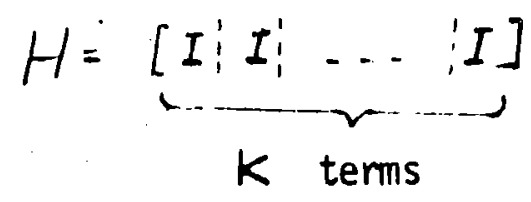


Since the "complete" data is Gaussian, and the relation between $\underline{X}(t)$ and $y(t)$ is linear, the results developed for the Linear-Gaussian case can be applied here. The detailed derivation is given in Appendix B. The resulting algorithm is:

E-step For $k=1,2, \ldots k$, compute:

$$
\underline{X}_{k}^{(n)}(t)=\underline{J}_{k}(t j \underline{\theta}(n))+\beta_{k}\left[\underline{y}(t)-\sum_{k=1}^{K} \underline{\theta}_{k}\left(t j \underline{\theta}_{k}^{(n)}\right)\right]
$$

M-step For $k=1,2, \ldots k$

$$
\operatorname{Min}_{\underline{\theta}_{k}} \int_{T_{i}}^{T_{f}}\left[\underline{x}_{k}^{(n)}(t)-\underline{I}_{k}\left(t ; \underline{\theta}_{k}\right)\right]^{*} Q^{-1}\left[X_{k}^{(n)}(t)-I_{k}\left(t ; \underline{\theta}_{k}\right)\right] d t \longrightarrow \underline{\theta}_{k}^{(n+1)}
$$

where the $\beta_{k}^{\prime \prime}$ are real-valued positive scalars satisfying

$$
\sum_{b=1}^{k} \beta_{k}=1
$$

In the discrete data case, the algorithm takes the form:

E-step Compute:

$$
\underline{X}_{b}^{(n)}\left(t_{i} ; \underline{U}_{h}\right)=\underline{y}_{k}\left(t_{i} ; \underline{\theta}_{b}^{(n)}\right)+\beta_{k}\left[\underline{y}\left(t_{i}\right)-\sum_{k=1}^{K} \underline{J}_{k}\left(t_{i j} \underline{\theta}_{k}^{(n)}\right)\right]
$$

$\underline{\text { M-step }}$

$$
\operatorname{Min}_{\theta_{k}} \sum_{i=1}^{N}\left[\underline{X}_{k}^{(n)}\left(t_{i j} \underline{\theta}_{k}\right)-\underline{J}_{k}\left(t_{i j} \theta_{k}\right)\right]^{*} Q^{-1}\left[\underline{X}_{k}^{(n)}\left(t_{i}\right)-\underline{J}_{k}\left(t_{i j} \underline{\theta}_{k}\right)\right] \longrightarrow \underline{\theta}_{k}^{(n+1)}
$$

The optimization required by (16.2) is, in fact, the optimization problem associated with the ML estimation of the $\quad \boldsymbol{\theta}_{k} \quad$ given separate observations of the $I_{k}\left(t ; \underline{\theta}_{6}\right)$. An entirely parallel statement can be made with respect 
to the optimization required by (17.2). Thus, the algorithm decouples the optimization associated with the direct ML approach into the $K$ separate $M L$ optimizations as illustrated in Fig. 1. Hence, the complexity of the algorithm is completely unaffected by the assumed number of signal components. As $K$ increases, we have to increase the number of ML processors in parallel; however, each processor is maximized separately. Since the algorithm is based on the EM method, it must converge to the exact ML estimate of the various $\underline{\theta}_{k}$ 's simultaneously, where each iteration increases the likelihood.

We note that the $\beta_{k}^{\prime} s$ must satisfy the constraint (16.3), but otherwise they are arbitrary free variables in the algorithm. The choice of the $\beta_{k}$ 's does not affect the value of the final estimates; however, they can be used to control the rate of convergence of the algorithm.

\section{Gaussian Signals}

Consider the model of (10) under the following assumptions:

- The $\underline{1}_{k}\left(t ; \underline{\theta}_{k}\right) \quad k=1,2, \ldots k$ are mutually uncorrelated, wide sense stationary (WSS), zero-mean Gaussian vector stochastic processes with the spectral density matricies $S_{k}\left(\omega_{j}, \theta\right) k=1,2, \ldots K$ respectively.

- $\quad(t)$ is uncorrelated. WSS, zero-mean and Gaussian with the spectral density matrix $N(\omega)$.

If the observation interval $T=T_{f}-T_{i}$ is long compared with the correlation time (inverse bandwidth) of the signals and the noises, the log-likelihood is given by (see Appendix $c$ )

$$
\log f_{\underline{Y}}(\underline{\underline{y}} ; \underline{\theta})=-\sum_{l}\left[\log d e t P\left(\omega_{l} ; \underline{\theta}\right)+\underline{Y}\left(\omega_{l}\right) P^{-1}\left(\omega_{l} ; \underline{\theta}\right) Y\left(\omega_{l}\right)\right]
$$

where 


$$
Y\left(\omega_{l}\right)=\frac{1}{\sqrt{T}} \int_{T_{i}}^{T_{f}} \underline{y}(t) e^{-j \omega_{l} t} d t \quad \omega_{l}=\frac{2 \pi}{T} \cdot l
$$

and

$$
P(\omega ; \underline{\theta})=\sum_{k=1}^{K} S_{k}\left(\omega j \underline{\theta}_{k}\right)+N(\omega)
$$

The sumation in (18.1) is carried over all $\omega_{l}$ in the signal frequency band.

In the case of discrete observations $y_{i}=y(i \cdot \Delta t)$, the log-likelihood is still given by $(18.1)$, where $\left\{\underline{Y}\left(\omega_{l}\right)\right\}_{l=0}^{N-1} \quad \omega_{l}=2 \pi l / N$ are the discrete Fourier transform (DFT) of $\left\{\underline{y}_{i}\right\}_{i=0}^{N-1}$, and $P(\omega ; \theta)$ is given by (18.3), where $S_{k}\left(\omega_{j} \underline{\theta}_{k}\right)$ is the spectral matrix of the corresponding sampled signal, and $N(\omega)$ is the spectral matrix of the sampled noise. Thus, in either case, the joint ML estimation of the various $\theta_{k}$ ' $_{j}$ is obtained as the solution to:

$$
\begin{gathered}
\operatorname{Min}_{\underline{\theta}_{1}, \underline{v}_{1}, \ldots \underline{\theta}_{k}}\left\{\sum_{l}\left[\log \operatorname{det} \rho\left(\omega_{l} ; \underline{\theta}\right)+\underline{Y}^{*}\left(\omega_{l}\right) P^{-1}\left(\omega_{l} ; \underline{\theta}\right) \underline{y}\left(\omega_{l}\right)\right]\right\} \\
\longrightarrow \hat{\theta}_{1}, \hat{\theta}_{2}, \ldots \hat{\theta}_{k}
\end{gathered}
$$

Thus, as in the deterministic signal case, the direct ML approach requires the joint optimization of the objective function with respect to all the $\underline{\theta}_{k}^{\prime} \mathrm{g}$. Using the EM method, we would like to simplify the required optimization. Using the same definition for the "complete" data as in the deterministic signal case (Eq.(14)) and applying the results developed for the Linear-Gaussian case, we obtain the following algorithm: 
E-step Compute:

$$
\begin{aligned}
\Psi_{k}^{(n)}\left(\omega_{l}\right) & =B_{k}\left(\omega_{l} j \underline{\theta}_{k}^{(n)}\right)\left[I-P^{-1}\left(\omega_{l} ; \underline{\theta}^{(n)}\right) B_{k}\left(\omega_{l} j \underline{\theta}_{k}^{(n)}\right)\right]+ \\
& +B_{k}\left(\omega_{l} ; \underline{\theta}_{k}^{(n)}\right) P^{-1}\left(\omega_{l} ; \underline{\theta}^{(n)}\right) Y\left(\omega_{l}\right) Y^{*}\left(\omega_{l}\right) P^{-1}\left(\omega_{l} ; \underline{\theta}^{(n)}\right) B_{k}\left(\omega_{l} ; \underline{\theta}_{k}^{(n)}\right)
\end{aligned}
$$

M-step

$$
\operatorname{Min}_{\underline{\theta}_{k}} \sum_{l}\left[\log \operatorname{det} B_{k}\left(\omega_{l j} \underline{\theta}_{k}\right)+\operatorname{tr}\left(B_{k}\left(\omega_{j} \theta_{k}\right) \Psi_{k}^{(n)}\left(\omega_{l}\right)\right)\right] \Longrightarrow \theta_{k}^{(n+1)}
$$

where we define

$$
B_{k}\left(\omega ; \underline{\vartheta}_{k}\right)=S_{k}\left(\omega j \underline{\theta}_{k}\right)+\beta_{k} \cdot N(\omega)
$$

and the $\beta_{b}{ }^{\prime} s$ are arbitrary real-valued scalars satisfying the condition (16.3). The detailed considerations leading to Eq.(20) are given in Appendix D. Perhaps the most striking feature of the algorithm is that it decouples the complex optimization associated with the direct ML approach into the $K$ separate ML optimizations as illustrated in Fig. 1: Thus, as in the deterministic signal case, we obtain a considerable simplification in estimator structure and computations.

\section{Example: Multiple Source Location Estimation}

The basic system of interest here consists of several spatially distributed signal sources and an array. of several spatially distributed sensors. The observed signals can be modelled using $(10)$, where $\varrho_{k}\left(t_{j} \vartheta_{k}\right)$ are the array signals received 
from the $k$ source, and $\underline{\theta}_{k}$ are the source location parameters (bearing and range, velocity components, etc.). Using the proposed scheme (Fig. 1), we obtain the exact ML estimates of the various source location parameters, while circumventing the complex multiple parameter optimization associated with the direct ML approach. For more details we refer the interested reader to 14$]$ and $[5]$. To simplify the example, we have considered the following situation:

- The array consists of 10 co-linear and evenly spaced sensors. The observed data consists of 100 time samples at each sensor output.

- There are three far-field sources at bearings $0,0.15$ and 0.3 radians, measured relative to the Boresight direction. Each source radiates a triangular pulse whose power (energy per sample) is normalized to 1 , and whose duration is 20 time points.

- The additive noises are uncorrelated from sensor to sensor with the same spectral level of 0.5 (that is, the post-integration SNR per channel is approximately $16 \mathrm{db})$.

We have also assumed that $\Delta L / U \cdot \Delta t=10$, where $\Delta t$ is the sampling period, $\Delta L$ is the spacing between adjacent sensors, and $U$ is the velocity of propagation in the medium. The problem is to estimate the various source bearings simultaneously.

In Fig. 2 we have plotted the conventional beam-former output as a function of bearing. As we can see, the standard method cannot resolve the various sources. In Fig. 3 we have plotted the outcome using our algorithm. We clearly see that after about 5 iterations, the algorithm essentially converges to the true bearings, and the three sources are resolved correctly. 
V. Conclusions

We have presented a computationally efficient scheme for parameter estimation of composite signals based on the EM algorithm. The proposed scheme is optimal in the sense that it converges iteratively to the exact Maximum Likelihood estimate of all the unknown parameters simultaneously. The method can be applied to a wide range of problems arising in signal and array processing. 
Appendix A The EM Algorithm

Let $H(X)=\underline{Y}$ where $H(\cdot)$ is a non-invertable (many-to-one) transformation. Express densities

$$
f_{\underline{X}}(\underline{x} ; \underline{\theta})=f_{\underline{V}}(H(\underline{x}) ; \underline{\theta}) \cdot f_{\underline{X} / \underline{Y}=\underline{y}}(\underline{\underline{x}} ; \underline{\theta})
$$

Taking the logarithm on both sides of (A.1), we obtain

$$
\log f_{y}(H(y) ; \underline{\theta})=\log f_{\underline{x}}(\underline{x} ; \underline{\theta})-\left.\log \right|_{x / y=\underline{y}}(\underline{x} ; \underline{\theta})
$$

Denote $\underline{y}=H(x)$ and taking conditional expectations given $\underline{Y}=\underline{y}$ for a parameter value $\underline{\theta}^{\prime}$, we obtain

$$
\begin{aligned}
& \log / \underline{y}(\underline{y} ; \underline{\theta})=E\{\log / \underline{x}(\underline{x} ; \underline{\theta}) / Y=\underline{y} ; \underline{\theta} 1\}- \\
& -E\left\{\log \left(x / y=\underline{y}(\underline{x}, \theta) / y=\underline{y} ; \underline{\theta}^{\prime}\right\}=U\left(\underline{\theta}, \underline{\theta}^{\prime}\right)-V\left(\underline{\theta}, \underline{\theta}^{\prime}\right)\right.
\end{aligned}
$$

Invoking the Jensen's inequality, we have that $V\left(\underline{\theta}, \underline{\underline{\theta}}^{\prime}\right) \leq V\left(\underline{\theta}^{\prime}, \underline{\theta}^{\prime}\right)$. Hence

$$
U\left(\underline{\theta}, \underline{\theta}^{\prime}\right)>U\left(\underline{\theta^{\prime}}, \underline{\theta}^{\prime}\right) \longrightarrow \log { }_{y}(\underline{y} ; \underline{\theta})>\left.\log \right|_{y}\left(\underline{y} ; \underline{\theta}^{\prime}\right)
$$

Eq.(A.4) forms the basis to the EM algorithm. The algorithm starts with an initial guess $\underline{\theta}^{(0)}$, and let $\underline{\theta}^{(n+1)}$ be defined inductively by

$$
\operatorname{Max}_{\underline{\theta}} U\left(\underline{\theta}, \underline{\theta}^{(n)}\right) \longrightarrow \underline{\theta}^{(n+1)}
$$


Eqs. (A.5) and (3) are identical. We note that since $\underline{\theta}(n+1)$ is the value of $\underline{\theta}$ that maximizes $U\left(\underline{\theta}, \underline{\theta}^{(n)}\right)$, then according to $(A .4)$, each iteration of the algorithm increases the value of $\log / \underline{y}(\underline{y} ; \underline{0})$. 
$-18-$

Appendix B. Derivation of Eq.(16)

The "complete" data $\underline{X}(t)$ is given by

$$
\underline{x}^{*}(t)=\left(\underline{x}_{1}^{*}(t) ! \underline{x}_{2}^{*}(t) ! \ldots ! \underline{x}_{k}^{*}(t)\right)
$$

where the $\underline{x}_{k}(t)$ are given by $(14.2)$, rewritten here for reference

$$
\underline{X}_{k}(t)=\underline{I}_{k}\left(t ; \underline{\theta}_{k}\right)+\underline{n}_{k}(t)
$$

The $\underline{n}_{k}(t)$ are chosen to be statistically independent, zero-mean and Gaussian with the covariance $E\left\{\underline{n}_{k}(t) \underline{n}_{k}^{*}(\Delta)\right\}=Q_{k} \cdot \Gamma(t-\Delta)$, where $Q_{k}=\beta_{k} \cdot Q$. The $\underline{x}_{k}(t)$ are, therefore, mutually independent and hence, following the usual considerations ([3], Chap. 4), we obtain

$$
\begin{aligned}
& \log f_{\underline{x}}(\underline{x} ; \underline{\theta})=\sum_{k=1}^{K} \log f_{X k}\left(\underline{x}_{k} ; \underline{\theta}_{k}\right) \\
&=c-\sum_{k=1}^{K} \int_{T_{i}}^{T_{f}}\left[\underline{x}_{k}(t)-\underline{J}_{k}\left(t ; \underline{\theta}_{k}\right)\right]^{*} Q_{k}^{-1}\left[\underline{x}_{k}(t)-\underline{J}_{k}\left(t ; \underline{\theta}_{k}\right)\right] d t
\end{aligned}
$$

Thus,

$$
\begin{aligned}
& E\left\{\log / x(x ; \underline{x}) / y=\underline{y} j \underline{\theta}^{(n)}\right\}= \\
& =c^{\prime}-\sum_{k=1}^{k} \int_{T_{i}}^{T_{f}}\left[\underline{x}_{k}^{(n)}(t)-\underline{I}_{k}\left(t_{j} \underline{\theta}_{k}\right)\right]^{*} Q_{k}^{-1}\left[\underline{x}_{k}^{(n)}(t)-I_{k}\left(t j \underline{\theta}_{k}\right)\right] d t
\end{aligned}
$$

where $c^{\prime}$ contains all the terms that are independent of $\underline{\theta}$. The $\underline{x}_{k}^{(n)}(t)$ are the components of. $\left.X^{(n)} / t\right)$, to be computed from 


$$
\underline{x}^{(n)}(t)=E\left\{\underline{x}(t) / H \cdot \underline{x}(t)=\underline{y}(t) ; \underline{\theta}^{(n)}\right\}
$$

Using Eq.(7) in (B.4) and following some straightforward matrix manipulations, we obtain (16.1). Now, since $\underline{\theta}_{k}$ enters the right side of (B.4) only through the $k$ term in the surn, the joint maximization of (B.3) with respect to the various $\underline{\theta}_{k}^{\prime} / \mathcal{A}$ decouples into the $K$ separate optimizations as suggested by (16.2). 
$-20-$

Appendix C. Derivation of Eq.(18)

Let the data vector $\underline{Y}$ be denerated by Fourier analyzing $y(t)$, that is

$$
\underline{Y}^{*}=\left(\underline{Y}^{*}\left(\omega_{1}\right) ; \underline{Y}^{*}\left(\omega_{2}\right): \ldots .\right)
$$

where the $Y\left(\omega_{l}\right)$ are defined by $(18.2)$. Since $y(t)$ is Gaussian and the $Y(\omega)$, are obtained by performing linear operations on $y / t)$, then the $Y\left(\omega_{l}\right)$ are jointly Gaussian. Since both signals and noises are assumed to be WSS, then for observation interval $T$ long compared with the correlation time of the signals and the noises, the $Y\left(\omega_{l}\right)$ are mutually uncorrelated (and thus, in the Gaussian case, statistically independent) with the covariance matrix given by

$$
E\left\{Y\left(\omega_{l}\right) Y^{*}\left(\omega_{l}\right)\right\}=P\left(\omega_{l} ; \underline{\theta}\right)
$$

where $P\left(\omega_{l}, \theta\right)$ is defined by (18.2). It follows that

$$
l_{\underline{Y}}\left(\underline{\underline{y} ; \underline{\theta})}=\prod_{l} \frac{1}{\operatorname{det} P\left(\omega_{l} ; \underline{\theta}\right)} e^{-\underline{Y\left(\omega_{l}\right)} P\left(\omega_{l} ; \underline{\theta}\right) \underline{Y}\left(\omega_{l}\right)}\right.
$$

Taking the logarithm on both sides of (C.3) immediately yields (18.1). 
Appendix $0 . \quad$ Derivation of Eq. (20)

Let the "complete" data $\underline{X}$ be generated by Fourier analyzing the various $\underline{x}_{k}(t)$, that is

where

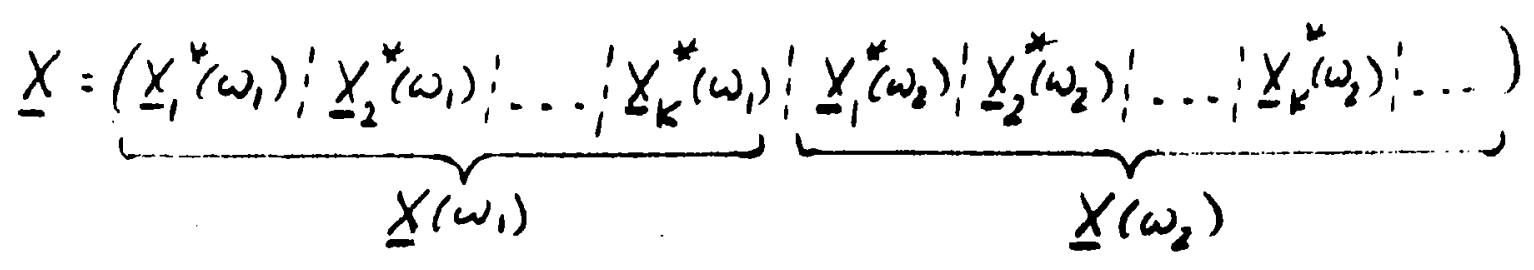

$$
\underline{X}_{k}\left(\omega_{l}\right)=\frac{1}{\sqrt{T}} \int_{T_{i}}^{T_{f}} X_{k}(t) e^{-j \omega_{l} t} d t
$$

and $\underline{x}_{k}(t)$ are given by $(14.2)$, rewritten here for reference

$$
\underline{X}_{k}(t)=\underline{J}_{k}\left(t ; \underline{\theta}_{k}\right)+\underline{n}_{k}(t)
$$

The $\underline{Y}_{k}(t)$ are chosen to be statistically independent, zero-mean and Gaussian with the spectral density matrix $N_{k}(\omega)=\beta_{k} \cdot N(\omega)$. Since the $\underline{I}_{k}\left(t_{j} \underline{\theta}_{k}\right)$ are assumed to be statistically independent, then the $\underline{X}_{k}(t)$ are statistically independent, in which case $\underline{X}_{k}\left(\omega_{e}\right)$ and $\underline{X}_{m}\left(\omega_{\beta}\right)$ are statistically independent whenever $k \neq m$. Furthermore, for long observation intervals and WSS processes, the $\underline{X}_{k}\left(\omega_{l}\right)$ at different frequencies are statistically uncorrelated (and thus independent) with the covariance matrix

$$
E\left\{X_{k}\left(\omega_{l}\right) \underline{x}_{k}^{*}\left(\omega_{l}\right)\right\}=B_{k}\left(\omega_{l j} \theta_{k}\right)
$$

where $B_{k}\left(\omega_{j} \underline{\theta}_{k}\right)$ is defined by (20.3). It follows that 
$-22-$

$$
f_{\underline{x}}\left(\underline{x} ; \underline{\underline{\partial})}=\prod_{k=1}^{k} \prod_{l} \frac{1}{\operatorname{det} B_{k}\left(\omega_{l} ; \underline{\theta}_{l}\right)} e^{-\underline{x}_{k}^{*}\left(\omega_{l}\right) B_{k}^{-1}\left(\omega_{l} ; \underline{\theta}_{k}\right) \underline{X}_{k}\left(\omega_{l}\right)}\right.
$$

Taking the logarithm on both sides of $(D .5)$, we obtain

$$
\log f_{\underline{x}}(\underline{x} ; \underline{\underline{\theta}})=-\sum_{k=1}^{k} \sum\left[\log \operatorname{det} B_{k}\left(\omega_{l} ; \underline{\theta}_{k}\right)+\underline{X}_{k}^{*}\left(\omega_{l}\right) B_{k}^{-1}\left(\omega_{l} j \underline{\theta}_{k}\right) \underline{X}_{k}\left(\omega_{l}\right)\right]
$$

Taking the conditional expectation given $Y=y$ for a parameter value $\underline{\theta}^{(n)}$, we obtain

$$
\begin{gathered}
E\left\{\log \mid \underline{x}(\underline{\underline{x}} ; \underline{\theta}) / \underline{y}=\underline{y}_{j} \underline{\underline{\theta}}(n)\right\}= \\
=\sum_{k=1}^{K} \sum_{l}\left[\log \operatorname{det} B_{k}\left(\omega_{l} ; \underline{\theta}_{k}\right)+\operatorname{tn}\left(B_{k}^{-1}\left(\omega_{l} ; \underline{\theta}_{k}\right) \Psi_{k}^{(n)}\left(\omega_{l}\right)\right)\right]
\end{gathered}
$$

where $\Psi_{k}^{(n)}\left(\omega_{l}\right)$ is the $(k, k)$ block matrix of $\Psi^{(n)}\left(\omega_{l}\right)$, to be computed from

$$
\Psi^{(n)}\left(\omega_{l}\right)=E\left\{\underline{X}\left(\omega_{l}\right) \underline{X}\left(\omega_{l}^{*}\right) / H \underline{X}\left(\omega_{l}\right)=\underline{Y}\left(\omega_{l}\right) ; \underline{g}^{(n)}\right\}
$$

Using Eq.(8) in (0.8) and following some straightforward matrix manipulations, we obtain (20.1). Since the $(k, l)$ term of the double sum on the right side of $(0.7)$ depends only on $\underline{\theta}_{k}$, then the joint maximization of (D.7) with respect to the various $\underline{\theta}_{6}$ decouples into the $K$ separate optimizations as suggested by (20.2). 


\section{References}

[1] A.P. Dempster, N.M. Laird and D.B. Rubin, "Maximum Likel ihood from Incomplete Data Via the EM Algorithm", Ann. of the Royal Stat. Soc., pp. 1-38, Dec. 1977.

[2] B.D.0. Anderson and J.B. Moore, Optimal Filtering, Prentice-Ha11, Inc. N.J. 1979.

[3] H.L. Van-Trees, "Detection, Estimation and Modulation Theory", Part I, Wiley, N.Y. 1968.

[4] M. Feder and E. Weinstein, "Optimal Multiple Source Location Estimation Via the EM Algorithm", Proc. of the 1985 Int. Conf. on Acoust., Speech \& Signal Processing (ICASSP'85), Vol. 4, pp. 1762-5, March 1985.

[5] M. Feder and E. Weinstein, "Multipath and Multiple Source Array Processing Via the EM Algorithm", Proc. of the 1986 Int. Conf. on Acoust., Speech \& Signal Processing (ICASSP'86). 


\section{Figure Captions}

Fig. 1: Signal Processing Via the EM Algorithm.

Fig. 2: Conventional Beamforming.

Fig. 3: Multiple Source Location Estimation Via the Proposed Algorithm. 


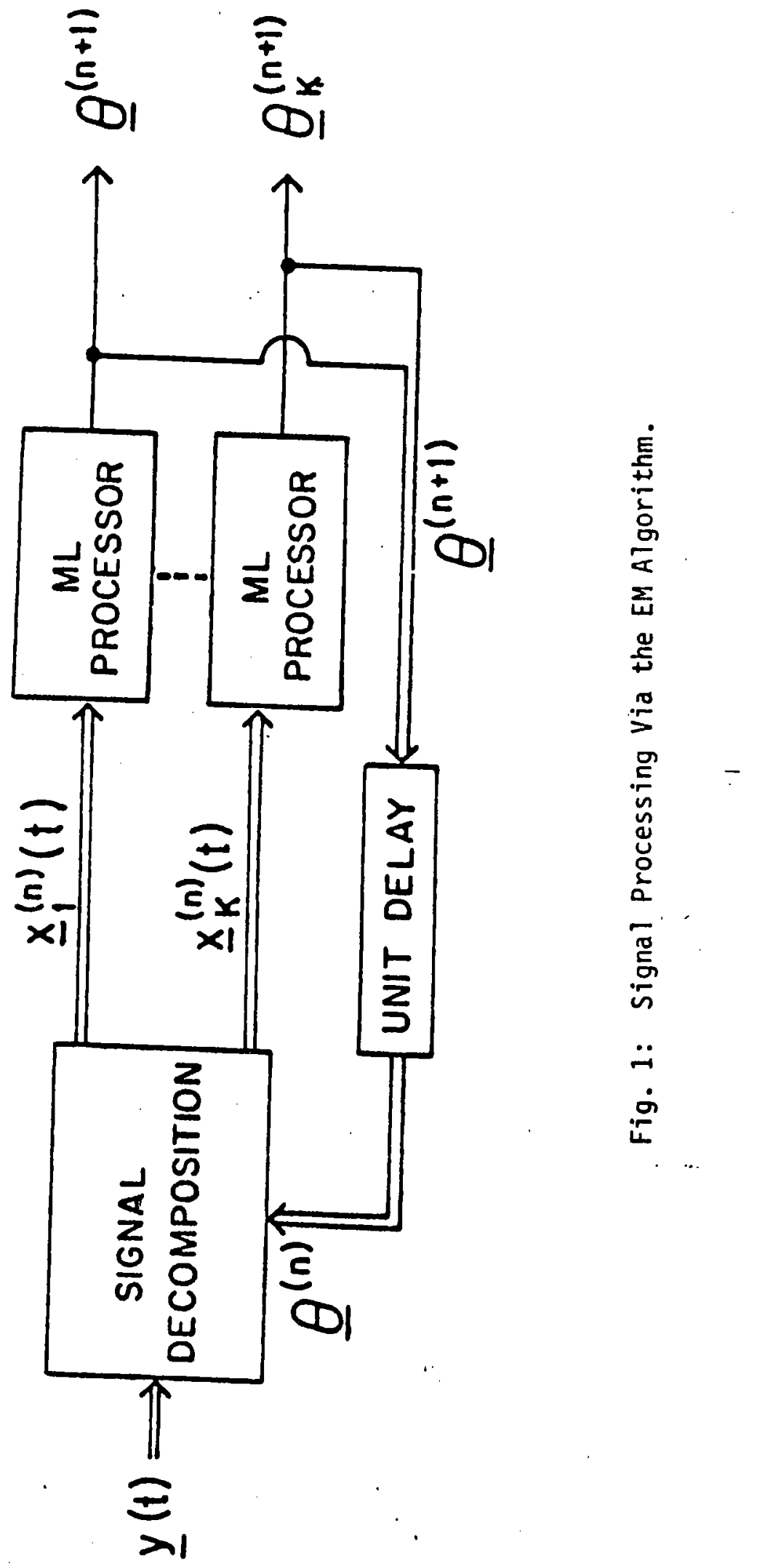


$-26-$

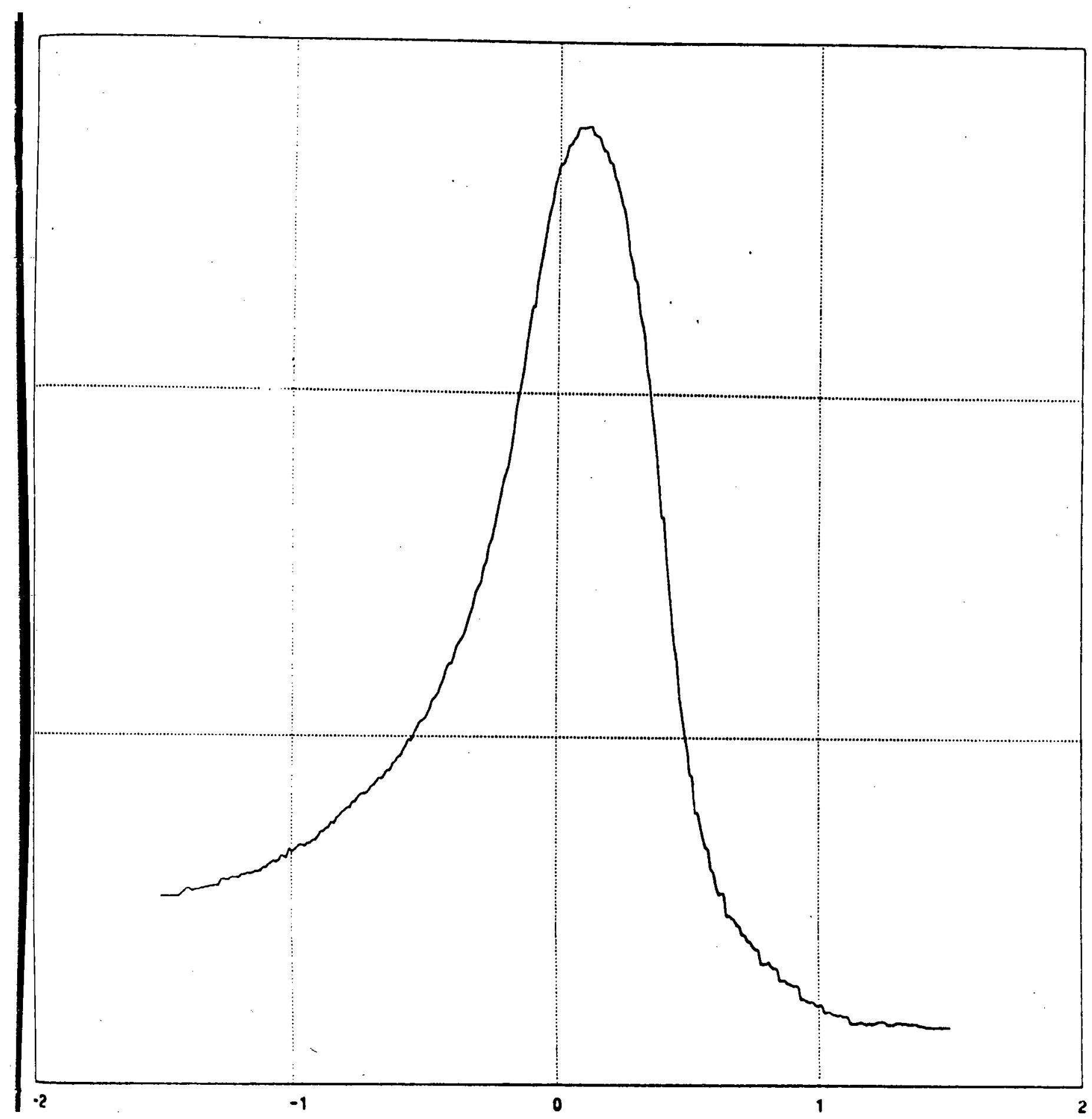

Fig. 2: Conventional Beamforming. 


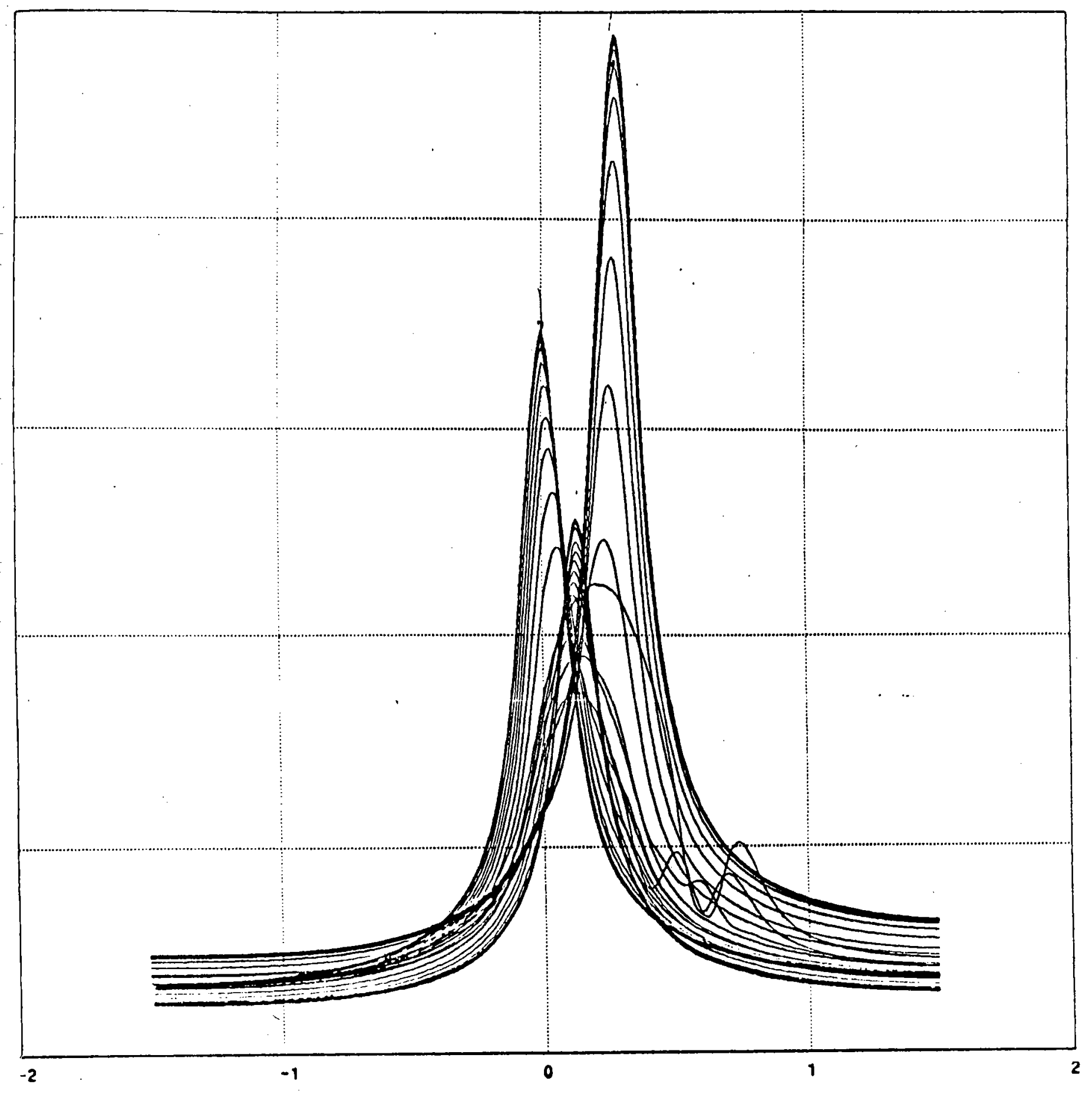

Fig. 3: Multiple Source Location Estimation Via the Proposed Algorithm. 


\section{DOCUMENT LIBRARY}

April 9, 1985

\section{DISTRIBUTION LIST FOR TECHNICAL REPORT EXCHANGE}

Institute of Marine Sciences Library

University of Alaska

O'Neill Building

905 Koyukuk Ave., North

Fairbanks, AK

Attn: Stella Sanchez-Wade

Documents Section

- Scripps Institution of Oceanography

Library, Mail Code C-075C

La Jolla, CA 92093

Hancock Library of Biology \& Oceanography

Alan Hancock Laboratory

University of Southern California

University Park

Los Angeles, CA 90089-0371

Gifts \& Exchanges

Library

Bedford Institute of Oceanography

P.O. Box 1006

Dartmouth, NS, B2Y 4A2, CANADA

Office of the International

Ice Patrol

c/o Coast Guard R \& D Center

Avery Point

Groton, CT 06340

Library

Physical Oceanographic Laboratory

Nova University

8000 N. Ocean Drive

Dania, FL 33304

NOAA/EDIS Miami Library Center 4301 Rickenbacker Causeway

Miami, FL 33149

\section{Library}

Skidaway Institute of Oceanography

- P.O. Box 13687

Savannah, GA 31416

- Institute of Geophysics

University of Hawaii

Library Room 252

2525 Correa Road

Honolulu, HI 96822

Library

Chesapeake Bay Institute

4800 Atwell Road

Shady Side, MD 20876
MIT Libraries

Serial Journal Room 14E-210

Cambridge, MA 02139

Director, Ralph M. Parsons Laboratory

Room 48-311

MIT

Cambridge, MA 02139

Marine Resources Information Center Bldg. E38-320

MIT

Cambridge, MA 02139

Library

Lamont-Doherty Geological Observatory

Colombia University

Palisades, NY 10964

Library

Serials Department

Oregon State University

Corvallis, OR 97331

Pell Marine Science Library

University of Rhode Island

Narragansett Bay Campus

Narragansett, RI 02882

Working Collection

Texas A\&M University

Dept. of Oceanography

College Station, TX 77843

Library

Virginia Institute of Marine Science

Gloucester Point, VA 23062

Fisheries-Oceanography Library

151 Oceanography Teaching Bldg.

University of Washington

Seattle, WA 98195

Library

R.S.M.A.S.

University of Miami

4600 Rickenbacker Causeway

Miami, FL 33149 


\section{Multiple Source Locetion Estimation Using the EM Algorithm}

7. Author(s)

Ehud Weinstein and Meir Feder

9. Parforming Organization Name and Addrese Woods Hole Oceanographic Institution

Woods Hole, Massachusetts 02543
Woods Hole, Massachusetts 02543

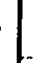

12. Sponsorine Orcanization Neme and Address

Naval Air Systems Command

15. Supplementery Notes

This report should be cited as: Woods Hole Oceanog. Inst. Tech. Rept. WHOI-86-26.

16. Abstract (Limit: 200 words)

We present a computationally efficient scheme for multiple source location estimation based on the EM Algorithm. The proposed scheme is optimal in the sense that it converges iteratively to the exact Maximum Likelihood estimate of all the unknown parameters simultaneously. The method can be applied to a wide range of problems arising in signal and array processing.

17. Document Analysis Descripton
1. Array Processing
2. Multiple Source Localization
3. EM Algorithm

b. Identifiers/Open-Ended Terms

c. Cosati FIald/Group

18. Avallobility Stetemen:

Approved for publication; distribution unlimited.

\begin{tabular}{|c|c|}
\hline $\begin{array}{l}\text { 19. Socurtiy Clases (This Roport) } \\
\text { UNCLLASSIFIED }\end{array}$ & $\begin{array}{l}\text { 21. No. of Peace } \\
27\end{array}$ \\
\hline 20. Socurtity Clesse (This Paeso) & 22. Price \\
\hline
\end{tabular}

